\title{
The Design and Realization of Team Map Based on LBS
}

\author{
Xiao Luo \\ Department of Information Engineering \\ Beijing Institute of Fashion Technology \\ Beijing, China \\ E-mail: lxiaorow@sohu.com
}

\author{
Fei Guo, Yanping Wang \\ Department of Information Engineering \\ Beijing Institute of Fashion Technology \\ Beijing, China
}

\begin{abstract}
With the application of GIS, the mobile positioning and wireless communications technology, the location-based services (LBS) are increasingly widespread. Location-based services are a kind of value-added services. Mainly through GPS and WIFI mobile location technology, it can get the user's current location, provide users with location-related services and achieve the needs of users finally.

The main object of this paper is the team map system based on LBS. This system can share your location; get teammates' location and other major functions. After it sends 'http' request to the server, it can post the team though the users' information, and the team is bound users, send your location in real time to the server, and then the server initiates the request again to obtain the location information from his teammates who want to find. The system sets up under Win7 system server environment, the team map has developed by using Xcode environment under MacOS system. After the testing, the system is practical, simple and convenient to use, it has highly practical value.
\end{abstract}

Keywords_LBS; Team Map; MacOS; Post

\section{INTRODUCTION}

With the widely applications of GIS, wireless communications and mobile positioning technology and the Internet enhance the global communications. Location-based services (LBS) [1] are increasingly being used. Location-based services, it is through mobile telecommunications operator radio communications network (such as the GSM network, CDMA network [2]) or external positioning mode (such as GPS) to obtain location information, and provide users with a value-added service of the corresponding. It has two meanings:

Firstly, the mobile device or the user's location is determined; secondly various types of location-related information services are provided. It can be called as a range of services related with the positioning system or the MPS-Mobile Position Services [3]. For example, the mobile phone users could be found the current location, and then get the name and address of a hotel, theater, libraries and gas stations within the range of $1 \mathrm{~km}$ [4]. So LBS complete the two functions of localization and service by using the Internet or wireless networks between fixed or mobile users.

LBS is made by the mobile communication network and computer network.[5] The mobile terminals send request to mobile communication networks by the gateway; the LBS service platform processes the user's request and current location; and returns the results to the user via the gateway. The mobile terminal may be a mobile phone, a personal digital assistant (Personal Digital Assistant, PDA[6]), handheld computer (Pocket PC), or a desktop computer (desktop PC) via the Internet communication. Service platform includes WEB server, location server and LDAP (Lightweight Directory Access Protocol [7]) server.

The main target of this paper is team system based on LBS. Users of the system is that they want to know the current location of people. By sending the team user's information to the server, the server initiates a location information request from each side.

\section{SYSTEM DESIGN}

The modular design of the system is divided into main, team and addressing modules. Function of each module design is shown in the following figure. See as Fig.1.

When the user bounds on the team interface though the 'http' by the nickname, you need to enter your nickname and teammates' nickname and send requests to the server to bind. After a successful bounding, they can locate their own position, get each other location, display the route on the map and provide 3 different ways. When a user bounds on the team interface by UDP, you need to enter your local IP and IP of other ones, so as to achieve the effect of a real-time transmission of data.

\section{A. Main Menu Module}

The functional requirements of the module are mainly reflected in UI (User Interface) design. The main menu has two functions. Each function of the UI control design needs to reflect the characteristics of concise and clear, to meet the needs of users.

\section{B. Team module}

This function module is the most important module of this system. The functional requirements of the module are mainly reflected in the logical structure of the team system design.

When users enter http through the binding nickname team interface, you need to enter your nickname and teammate nickname to the server to request binding, after the successful 
binding into the team interface, you can achieve their own positioning, access to each other's location and displayed on the map and provide from his position to the location of his teammates three ways to find ways.

When the user enters UDP through the binding IP team interface, you need to enter the IP of each side, so as to achieve a real-time data transmission effect.

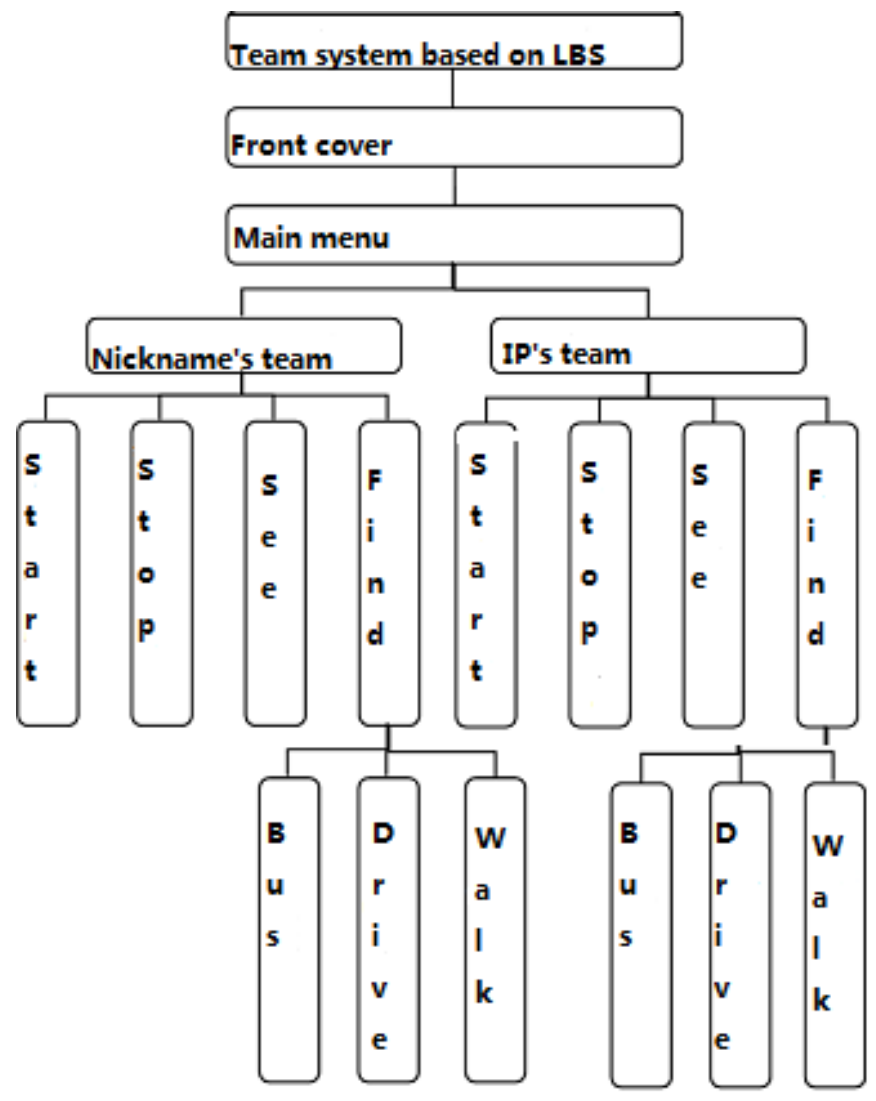

Fig. 1. Module function diagram

\section{THE SERVER DESIGN}

Server is a high-performance computer network environment. It listens to the submitted requests by the other computers (clients) on the network and provides the necessary services. In many cases, the computer runs through several servers to provide a variety of services. Server is running on the computer to meet other service requests. Therefore, the server performs some tasks on behalf of clients. Customers usually connected to the server through the network. In the Internet Protocol (IP) network, the server is a socket responder.

\section{A. XАMPP}

Login https://www.apachefriends.org/zh_cn/index.html; select the windows version of XAMPP to download.

Open XAMPP-win32-1.8.3-0-VC11-installer.exe
Running Apache and MySQL, build XAMPP. See as Fig.2 and Fig.3.

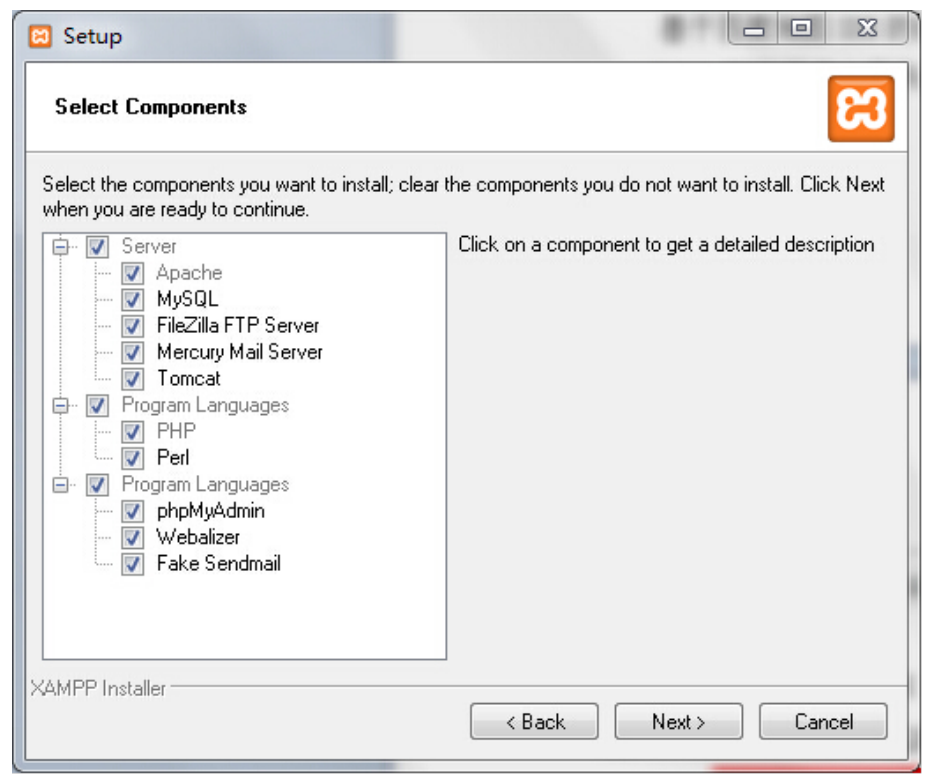

Fig. 2. XAMPP Set up

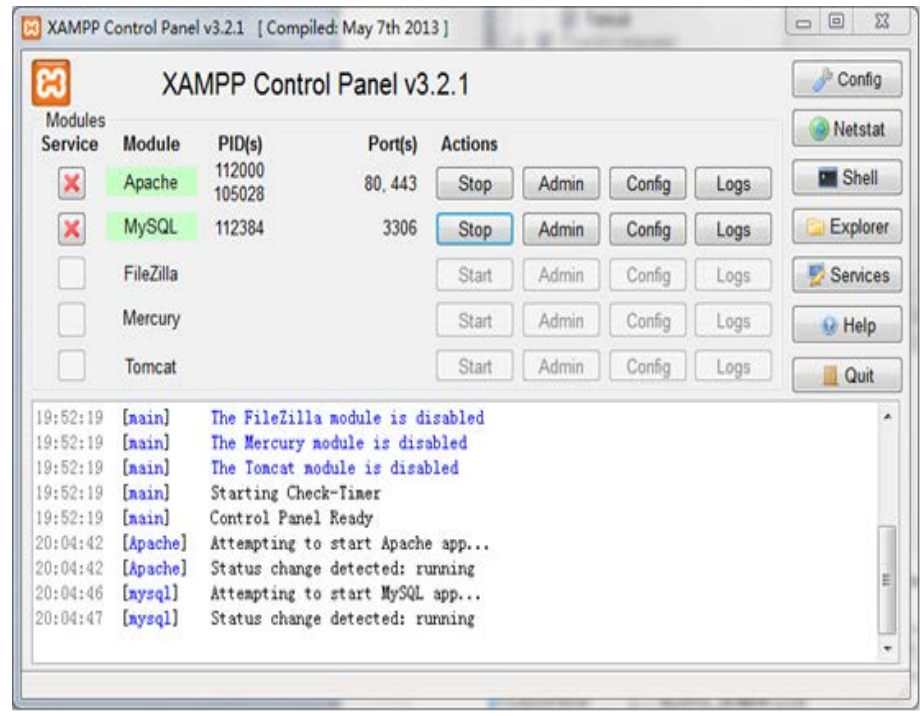

Fig. 3. XAMPP Configuration

\section{B. Navicat}

In order to manage mySQL, you need to install navicat, package, and make the new connection.

Create a database, named Team map, set the encoding format as utf8 - UTF-8 Unicode. Setting ID to the primary key, UID storage to device id (machine identification code for each device).

\section{Configure Server}

The database is exported to SQL file, and then to the Server.

The application server used by the team, detailed framework is shown below Fig.4. 


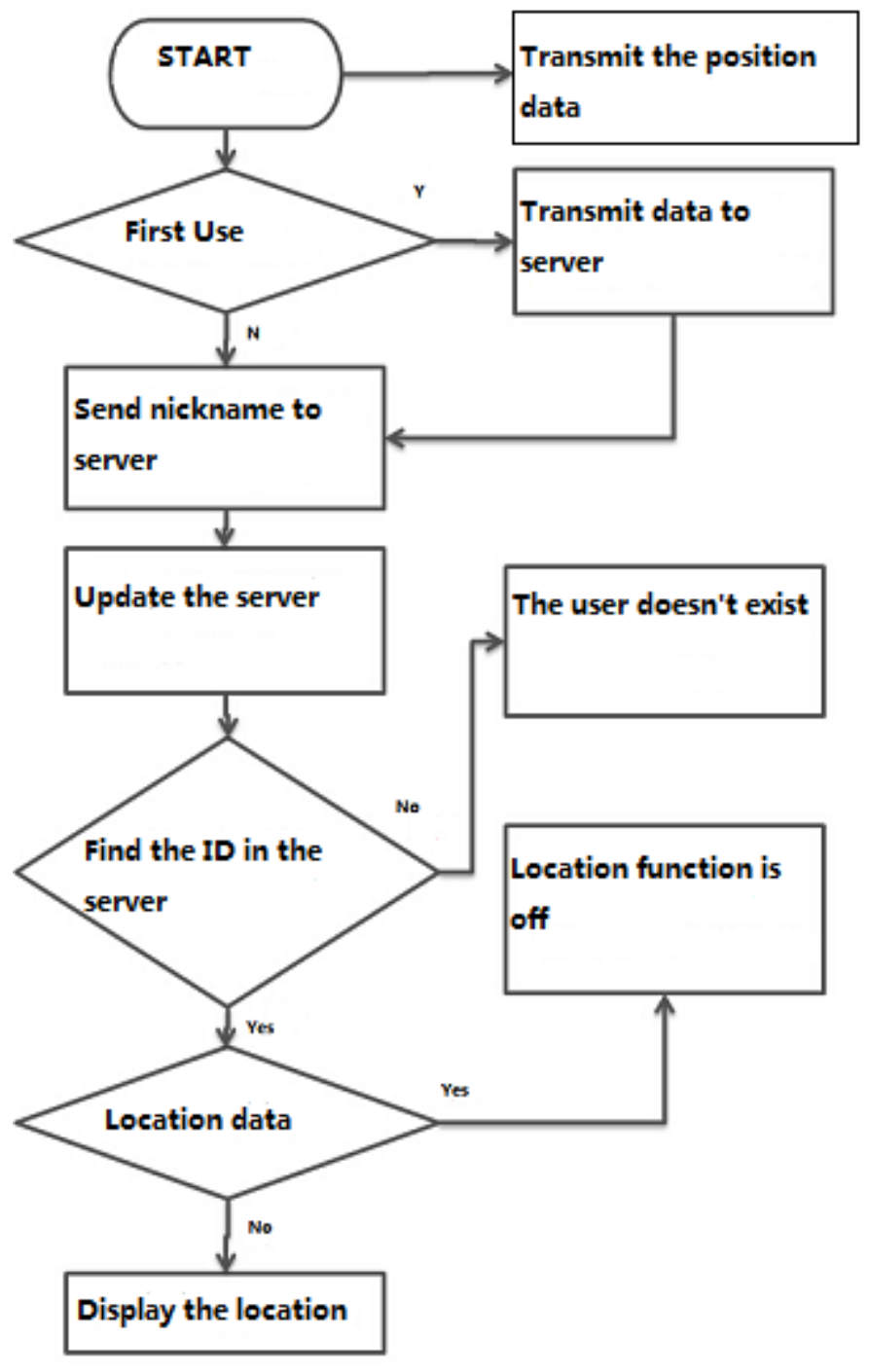

Fig. 4. Server detailed flow chart

- Post the user information to the server, which includes its nickname and teammates' nickname, the server gets the information and saves. It will be returned the flag of 'success' when the server has received the all data field.

- The user opens the positioning function to find their place on the map, and then sends the location information to the server in real time, including their own nicknames, longitude and latitude. If it is successful, the server returns 'success' field.

- When the user clicks the button to get the location information of teammates, it sends a request to the server post, including his nickname -'myuid'. When the server gets 'myuid', it finds the corresponding UID in the database, and returns the position of the UID to the user. Therefore, the user can find the position of his teammates. Because the server lookup logical teammate location information is such that it is possible to achieve more than the team. The team will need to enter his own IP, the machines own IP can be displayed on your team page by using analytic functions. Each mobile is both a server and a client. The flowchart is as the follow Fig.5:

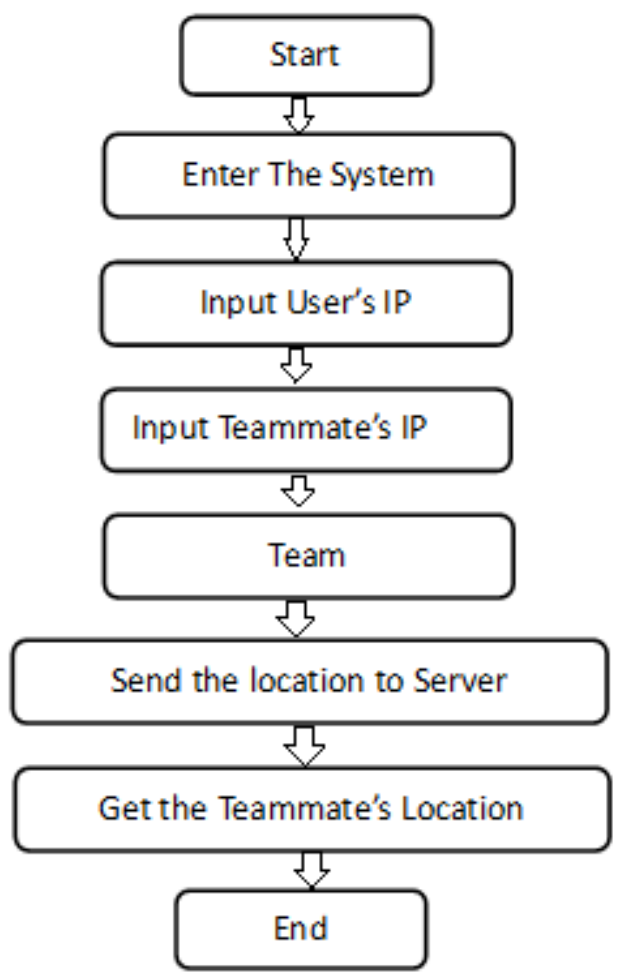

Fig. 5. Binding IP to team flowchart

\section{SYSTEM TESTING}

Software testing process can follow four steps to carry out, that is, unit testing, integration testing, validation testing and release testing [8][9].

\section{A. Unit testing:}

Testing each program unit with the source code, check each program module with correctly specified function.

\section{B. Integration testing}

Testing the main structure of the software.

\section{Validation testing}

Verify the specifications, configuration and the actual operating environment of software.

The detailed testing is as following Table 1. 
TABLE I. TEST AND MODIFY

\begin{tabular}{|c|c|c|}
\hline $\begin{array}{c}\text { Testing } \\
\begin{array}{c}\text { The interface of users' } \\
\text { information }\end{array}\end{array}$ & Result & How to modify \\
\hline $\begin{array}{c}\text { Server } \\
\text { Upload their own } \\
\text { location information } \\
\text { Interface }\end{array}$ & Pass & $\begin{array}{c}\text { B cannot receive the location of } \\
\text { A again when A, B is a team } \\
\text { and A, C is another one team. } \\
\text { Modify the database with new } \\
\text { ways, changed 'update' to } \\
\text { solve the conflict }\end{array}$ \\
\hline $\begin{array}{c}\text { Server } \\
\text { Get location information } \\
\text { teammate Interface }\end{array}$ & Not pass & $\begin{array}{c}\text { According UID the } \\
\text { nodify the 'select' statement, } \\
\text { first find the corresponding } \\
\text { teammate, and then find the } \\
\text { location information based on } \\
\text { ID }\end{array}$ \\
\hline Interface change & Pass & \begin{tabular}{c} 
nonding location of \\
\hline
\end{tabular} \\
\hline
\end{tabular}

\section{SUMMARY}

In this paper, a team map based on LBS system is discussed. The system design includes the main menu module, the module team, and wayfinding module. System test results show that the finished application meets the design requirements, with a highly practical, simple and convenient feature. However, the system still has some deficiencies, such as, the larger electricity consumption when locating and opening the application and the lack of two-dimensional code scanning bounding. These will gradually be improved in the subsequent amendments and further designs.

\section{ACKNOWLEDGMENT}

This research was financially supported by the BIFT research project NHFZ2016010 and NHFZ2016012.

\section{REFERENCES}

[1] HuLi Shi, Jun Pei. The solutions of navigation observation equations for CAPS[J]. Science in China Series G: Physics, Mechanics and Astronomy. 2009 (3)

[2] Federal Communications Commission. FCC Enhanced 911-wireless services. http://www.fcc.gov/911/enhanced/ . 2011

[3] Cheng J. S, Hsiang W. H, Wu W.C. The Design of Intelligent Mobile Tourism Service System. Proceedings of the 2010 International Computer Symposium . 2010

[4] Sarfraz Khokhar, Arne A. Nilsson.Introduction to Mobile Trajectory BasedServices: A New Direction in Mobile Location Based Services. Lecture Notesin Computer Science . 2009

[5] Anliang Ning, Fanshun Meng, Mingzhu Liu. Mobile Geographical Information Services for 3G Phone User. Wireless Communications, Networking and Mobile Computing, 2008.

[6] Robert P. Biuk-Aghai. A mobile GIS application to heavily resource-constrained devices[J]. Geo-spatial Information Science . $2004(1)$

[7] Andrew J. May, Tracy Ross,Steven H. Bayer, Mikko J. Tarkiainen. Pedestrian navigation aids: information requirements and design implications[J]. Personal and Ubiquitous Computing. 2003 (6)

[8] Klippel, A, Richter, K. F, Barkowsky, T \& Freksa, C.The cognitive reality of schematic maps. Map-Based Mobile Services-Theories, Methods and Implementations . 2005 (3)

[9] Sudhir Dixit, Tero Ojanpera,Richard van Nee, Ramjee Prasad. Introduction to Globalization of Mobile and Wireless Communications Today and in 2020. Signals and Communication Technology, 2011, Globalization of Mobile and Wireless Communications . 